Research Article

\title{
Clinical Evaluation of Pinggan Yiqi Yangshen Recipe Combined with Labetalol Hydrochloride and Magnesium Sulfate in the Treatment of PIH
}

\author{
Ping Li $\mathbb{D}^{1},{ }^{1}$ Jie Zhao $\mathbb{D}^{2},{ }^{2}$ Peipei Gao $\mathbb{D}^{3},{ }^{3}$ and Hongcui $Q u\left(\mathbb{D}{ }^{4}\right.$ \\ ${ }^{1}$ Health Education Department, Zhangqiu Maternity and Child Care Hospital, Jinan 250200, China \\ ${ }^{2}$ Outpatient Department, Zhangqiu Maternity and Child Care Hospital, Jinan 250200, China \\ ${ }^{3}$ Comprehensive Ward, Zhangqiu Maternity and Child Care Hospital, Jinan 250200, China \\ ${ }^{4}$ Outpatient Operating Room, Zhangqiu Maternity and Child Care Hospital, Jinan 250200, China
}

Correspondence should be addressed to Hongcui Qu; quhongcui@sdjnzqfy.cn

Received 6 September 2021; Accepted 18 October 2021; Published 28 October 2021

Academic Editor: Muhammad Wasim Khan

Copyright (C) 2021 Ping Li et al. This is an open access article distributed under the Creative Commons Attribution License, which permits unrestricted use, distribution, and reproduction in any medium, provided the original work is properly cited.

\begin{abstract}
Background. To observe the clinical effect of Pinggan Yiqi Yangshen recipe combined with labetalol hydrochloride and magnesium sulfate in the treatment of pregnancy-induced hypertension (PIH). Methods. A total of 126 patients with PIH diagnosed in our hospital from January 2016 to May 2018 were randomly divided into the control group and the experimental group, with 63 cases in each group. The control group was treated with labetalol combined with magnesium sulfate. On the basis of the control group, the experimental group was treated with Pinggan Yiqi Yangshen recipe. Clinical efficacy, blood pressure, renal function, and biochemical indexes were compared between the two groups. Moreover, pregnancy outcomes and adverse reactions were compared between the two groups. Results. After treatment, the total effective rate in the experimental group was higher than in the control group. Blood pressure and mean arterial pressure in the experimental group were more significantly downregulated than the control group. Renal function indexes and biochemical indexes in the experimental group were more significant than those in the control group. The incidence of cesarean section, preterm birth, and abnormal fetal heart rate in the experimental group was significantly lower than that in the control group. There was no difference in the incidence of fetal distress, postpartum hemorrhage, neonatal asphyxia, and adverse reactions between the two groups. Conclusion. Pinggan Yiqi Yangshen recipe combined with labetalol hydrochloride and magnesium sulfate can effectively reduce the blood pressure of patients with $\mathrm{PIH}$, help patients to return to normal levels of biochemical indexes and renal function indexes, and improve pregnancy outcomes with high safety, which is worthy of further promotion and application in clinical practice.
\end{abstract}

\section{Introduction}

Pregnancy-induced hypertension (PIH) is a disease that occurs more frequently in pregnant women. PIH usually refers to a pregnancy complication with hypertension, nausea, vomiting, edema, and proteinuria as the main clinical symptoms after 20 weeks of pregnancy $[1,2]$. If not treated timely, patients will suffer from convulsion, coma, heart failure, kidney failure, and other serious conditions and can also cause fetal distress, premature delivery, and even death. PIH seriously threatens the life safety of the mother and child and is one of the main causes of maternal death at present [3]. In recent years, the incidence of PIH has been increasing year by year [4], which has aroused great attention from doctors and patients. Clinically, western medicine is mainly used to treat patients with $\mathrm{PIH}$, among which magnesium sulfate and labetalol hydrochloride are the most common $[4,5]$. However, long-term use may lead to some adverse reactions in patients, such as weakened knee reflex and muscle weakness [6]. At present, western medicine combined with traditional Chinese medicine has been gradually used to treat $\mathrm{PIH}$, and its advantages have been reported in clinical practice. It can not only play the advantage of the rapid therapeutic effect of western medicine 
but also reflect the characteristics of the overall adjustment and treatment of both symptoms and root causes of traditional Chinese medicine [7, 8].

In this study, Pinggan Yiqi Yangshen recipe was used in combination with labetalol hydrochloride and magnesium sulfate to treat PIH in order to observe its clinical effect on $\mathrm{PIH}$ and the occurrence of adverse outcomes during pregnancy.

\section{Materials and Methods}

2.1. General Information. A total of 126 patients with PIH admitted to Zhangqiu Maternity and Child Care Hospital from January 2016 to May 2018 were randomly divided into the experimental group (63 cases) and control group (63 cases). There was no significant difference in general data between the two groups $(P>0.05$, Table 1$)$. The inclusion criteria were as follows: all patients met the diagnostic criteria of PIH in "Obstetrics \& Gynecology" [9]; all patients were singleton pregnancies; all patients were treated for the first time. The exclusion criteria were as follows: patients with serious heart, brain, kidney, liver, and other important viscera-related diseases; patients who are allergic to the treatment drugs in this study; patients who do not cooperate with the researcher. This study was approved by the ethics committee of Zhangqiu Maternity and Child Care Hospital, and all patients signed informed consent.

2.2. The Treatment. The patients in both groups were treated with 0.25-5g methyldopa (H11020968, China Resources Shuanghe Pharmaceutical Co., Ltd.), twice a day. The control group was treated with basic treatment combined with labetalol hydrochloride and magnesium sulfate. The usage and dosage of labetalol hydrochloride were as follows: the patients received an intravenous infusion of $50 \mathrm{mg}$ labetalol hydrochloride (H32026120, Jiangsu Desano Pharmaceutical Co., Ltd.) dissolved in $250 \mathrm{ml}$ of $5 \%$ glucose solution at $2 \mathrm{mg}$ / min, once a day, for 7 days. Severe patients were given $30 \mathrm{ml}$ $10 \%$ glucose solution containing $25 \mathrm{mg}$ labetalol hydrochloride by an intravenous drip. The usage and dosage of magnesium sulfate were as follows: the patients received an intravenous infusion of $60 \mathrm{ml}$ magnesium sulfate injection (national drug approval H33021961, Hangzhou Minsheng Pharmaceutical Co., Ltd.) dissolved in $500 \mathrm{ml} \mathrm{5 \%} \mathrm{glucose}$ injection at $1-2 \mathrm{~g} / \mathrm{h}$, once a day, for 7 days. The experimental group was added with Pinggan Yiqi Yangshen recipe on the basis of the control group. The prescription of Pinggan Yiqi Yangshen recipe was as follows: $15 \mathrm{~g}$ Eucommia ulmoides, $10 \mathrm{~g}$ Uncaria rhynchophylla, $30 \mathrm{~g}$ Astragali Radix, $20 \mathrm{~g}$ Semen Cassiae, $20 \mathrm{~g}$ Chrysanthemum morifolium, $15 \mathrm{~g}$ Poria cocos, $15 \mathrm{~g}$ Atractylodes macrocephala, $15 \mathrm{~g}$ Bupleurum chinense, 20 g Curcumae Radix, $30 \mathrm{~g}$ Radix Codonopsis, $10 \mathrm{~g}$ Rhizoma Alismatis, $6 \mathrm{~g}$ Pinelliae Rhizoma, $20 \mathrm{~g}$ Angelica, $15 \mathrm{~g}$ Angelica dahurica, $10 \mathrm{~g}$ Gardenia jasminoides, $10 \mathrm{~g}$ Citri Reticulatae Pericarpium, $10 \mathrm{~g}$ Cyperus rotundus, $10 \mathrm{~g}$ Paeoniae Alba Radix, 5 g Cortex Phellodendri, $15 \mathrm{~g}$ donkey-hide gelatin, and $15 \mathrm{~g}$ Herba Taxilli were decocted with water every morning and evening for 7 days, one course of treatment for light patients and two courses of treatment for severe patients.
2.3. Observation Indexes. The clinical effect of patients in two groups after treatment was observed. The criteria were as follows: (1) special effect: the patient's symptoms and signs were disappeared, the systolic blood pressure (SBP) was decreased $>30 \mathrm{mmHg}$, the diastolic blood pressure (DBP) was decreased $>20 \mathrm{mmHg}$, the blood pressure was normal, and $24 \mathrm{~h}$ urine protein (Upro) was decreased; (2) valid effect: the patients' symptoms and signs were disappeared or improved, SBP was decreased $<10 \mathrm{mmHg}$, DBP was decreased $<120 \mathrm{mmHg}$, blood pressure was basically normal, and Upro was decreased; (3) invalid effect: the patients' symptoms, signs, blood pressure, and Upro were not changed or worse. Total effective rate $=($ special + valid $) /$ total cases.

The improvement of blood pressure and mean arterial pressure before and after treatment was observed. The biochemical indexes of the two groups were detected. Biuret colorimetry (BT 2000 Plus Biochemical Analyzer, Italy BT) was used to detect the level of $24 \mathrm{~h}$ Upro. The level of superoxide dismutase (SOD) was measured by the xanthine oxidase assay (Xanthine Oxidase Test Kit, Shenzhen Zike Biotechnology Co., Ltd.). The plasma nitric oxide (NO) level was determined by nitric acid reductase (Nitric Oxide Test Kit, Shanghai Yisen Biological Technology Co., Ltd.). The level of malondialdehyde (MDA) was determined by the thiobarbituric acid method (Shanghai Mingbo Biological Technology Co., Ltd.). The plasma endothelin-1 (ET-1) level was determined by the radioimmunoassay (Shanghai Hengyuan Biological Technology Co., Ltd.). Renal function indexes were detected in both groups. The normal range of blood urea nitrogen (BUN) was 3.2-7.1 mmol/L; serum creatinine $(\mathrm{Cr})$ was $70-106 \mu \mathrm{mol} / \mathrm{L}$; serum uric acid (UA) was 89-357 $\mu \mathrm{mol} / \mathrm{L}$; serum urea (SU) was $1.78-7.14 \mathrm{mmol} / \mathrm{L}$. The adverse reactions and maternal and infant outcomes of the two groups were observed.

2.4. Statistical Analysis. SPSS 22.0 software was used to analyze the data. The measurement data was expressed as $\bar{x}+s$, and the counting data were expressed as $n(\%)$ and analyzed by $X^{2}$ test. $P<0.05$ was considered statistically significant.

\section{Results}

3.1. Comparison of Clinical Efficacy between the Two Groups. The total effective rate of the experimental group was $93.65 \%$, and that of the control group was $71.43 \%$. The clinical efficacy of the treatment group was significantly higher than that of the control group $\left(X^{2}=16.211, P<0.01\right.$, Table 2$)$.

3.2. Comparison of Renal Function Indexes and Biochemical Indexes in Two Groups. There was no significant difference in renal function and biochemical indexes between the two groups before treatment. After treatment, renal function indexes and biochemical indexes in both groups decreased compared with those before treatment, and the reduction in the experimental group was more significant than that in the control group $(P<0.01$, Tables 3 and 4$)$. 
TABLe 1: Comparison of general clinical data between the two groups.

\begin{tabular}{|c|c|c|c|c|}
\hline Clinical parameters & Experimental group $(n=63)$ & Control group $(n=63)$ & $X^{2}$ & $P$ value \\
\hline \multicolumn{5}{|l|}{ Age (years) } \\
\hline$\leq 32$ & 37 & 40 & \multirow{2}{*}{0.301} & \multirow{2}{*}{0.584} \\
\hline$>32$ & 26 & 23 & & \\
\hline \multicolumn{5}{|l|}{$B M I\left(\mathrm{~kg} / \mathrm{m}^{2}\right)$} \\
\hline$<18.5$ & 6 & 8 & \multirow{3}{*}{0.725} & \multirow{3}{*}{0.696} \\
\hline $18.5-24.9$ & 45 & 46 & & \\
\hline$\geq 25$ & 12 & 9 & & \\
\hline \multicolumn{5}{|c|}{ Gestational age (weeks) } \\
\hline$<28$ & 7 & 8 & \multirow{2}{*}{0.076} & \multirow{2}{*}{0.783} \\
\hline$\geq 28$ & 56 & 55 & & \\
\hline \multicolumn{5}{|l|}{ Parity } \\
\hline 1 & 41 & 39 & \multirow{2}{*}{0.137} & \multirow{2}{*}{0.711} \\
\hline$>1$ & 22 & 24 & & \\
\hline \multicolumn{5}{|l|}{ Severity degree } \\
\hline Mild & 8 & 9 & \multirow{3}{*}{0.469} & \multirow{3}{*}{0.791} \\
\hline Moderate & 49 & 50 & & \\
\hline Severe & 6 & 4 & & \\
\hline
\end{tabular}

TABLE 2: Comparison of clinical efficacy between the two groups.

\begin{tabular}{|c|c|c|c|c|c|}
\hline Group & $n$ & Special effect & Valid effect & Invalid effect & Total effective rate \\
\hline Experimental group & 63 & $57.14 \%(36 / 63)$ & $36.51 \%(23 / 63)$ & $6.35 \%(4 / 63)$ & $93.65 \%(59 / 63)$ \\
\hline Control group & 63 & $26.98 \%(17 / 63)$ & $44.44 \%(28 / 63)$ & $28.57 \%(18 / 63)$ & $71.43 \%(45 / 63)$ \\
\hline$X^{2}$ & & & & & 16.211 \\
\hline$P$ & & & & & $<0.01$ \\
\hline
\end{tabular}

TABLE 3: Comparison of biochemical indexes between two groups.

\begin{tabular}{lcccccc}
\hline \multicolumn{2}{c}{ Group } & NO $(\mathrm{mmol} / \mathrm{L})$ & SOD $(\mathrm{U} / \mathrm{ml})$ & $24 \mathrm{~h}$ Upro $(\mathrm{g})$ & $\mathrm{ET}-1(\mathrm{ng} / \mathrm{L})$ & $\mathrm{MDA}(\mathrm{nmol} / \mathrm{L})$ \\
\hline \multirow{2}{*}{ Experimental group } & Before treatment & $523.46 \pm 72.84$ & $63.21 \pm 8.73$ & $4.22 \pm 1.14$ & $93.56 \pm 17.73$ & $21.84 \pm 6.12$ \\
& After treatment & $971.43 \pm 104.57^{* \Delta}$ & $124.37 \pm 17.84^{* \Delta}$ & $1.25 \pm 0.82^{* \Delta}$ & $48.65 \pm 8.97^{* \Delta}$ & $10.76 \pm 4.11^{* \Delta}$ \\
\hline \multirow{2}{*}{ Control group } & Before treatment & $525.71 \pm 71.66$ & $61.28 \pm 8.16$ & $4.18 \pm 1.09$ & $94.11 \pm 18.06$ & $21.63 \pm 6.34$ \\
& After treatment & $747.23 \pm 79.47^{*}$ & $92.64 \pm 13.92^{*}$ & $2.71 \pm 0.94^{*}$ & $73.24 \pm 12.69^{*}$ & $15.93 \pm 4.45^{*} s$ \\
\hline$X^{2}$ & & 13.542 & 9.416 & 6.631 & 11.723 & 7.264 \\
\hline$P$ & & $<0.01$ & $<0.01$ & $<0.01$ & $<0.01$ & $<0.01$ \\
\hline
\end{tabular}

Compared with the same group before treatment, ${ }^{*} P<0.01$; compared with the control group after treatment, ${ }^{\Delta} P<0.01$.

3.3. Comparison of Blood Pressure and Mean Arterial Pressure in Two Groups. Before treatment, there was no significant difference in blood pressure between the experimental group and control group $(P>0.05)$. After treatment, SBP was $127.63 \pm 11.45 \mathrm{mmHg}$, DBP was $78.34 \pm 15.71 \mathrm{mmHg}$, and MAP was $101.66 \pm 6.23 \mathrm{mmHg}$. In the control group, SBP was $151.32 \pm 13.16 \mathrm{mmHg}$, DBP was $90.57 \pm 16.63 \mathrm{mmHg}$, and MAP was $121.37 \pm 7.54 \mathrm{mmHg}$. SBP, DBP, and MAP in both groups were lower than before treatment $(P<0.05)$, and the experimental group was more significantly decreased than the control group $(P<0.05$, Figures 1 and 2$)$.

3.4. Comparison of the Incidence of Adverse Reactions and Pregnancy Outcomes between the Two Groups. After treatment, the incidence of cesarean section, preterm birth, and abnormal fetal heart rate in the experimental group was significantly lower than that in the control group $(P<0.05)$. There was no significant difference in the incidence of fetal distress, postpartum hemorrhage, and neonatal asphyxia between the two groups $(P>0.05)$. There was no significant difference in the incidence of adverse reactions between the two groups $(P>0.05$, Tables 5 and 6$)$.

\section{Discussion}

$\mathrm{PIH}$ is caused by many factors, such as family genetic history, age, obesity, and hypertension. At the same time, insufficient nutritional intake and recurrent mood fluctuations during pregnancy can also lead to the occurrence of $\mathrm{PIH}[10,11]$. The main clinical manifestations of PIH are abnormally elevated blood pressure, proteinuria, edema, etc., accompanied by dizziness, nausea, systemic small vessel spasm, and other clinical symptoms. Furthermore, due to the high blood pressure of patients, there will be insufficient blood supply in the uterus and placenta, which will affect the uptake of oxygen and nutrients in the uterus of the fetus and be prone to fetal intrauterine growth retardation and other 
TABLE 4: Comparison of renal function indexes between two groups.

\begin{tabular}{|c|c|c|c|c|c|}
\hline \multicolumn{2}{|c|}{ Group } & $\mathrm{Cr}(\mu \mathrm{mol} / \mathrm{L})$ & $\mathrm{SU}(\mathrm{mmol} / \mathrm{L})$ & BUN $(\mathrm{mmol} / \mathrm{L})$ & $\mathrm{UA}(\mu \mathrm{mol} / \mathrm{L})$ \\
\hline Experimental group & $\begin{array}{l}\text { Before treatment } \\
\text { After treatment }\end{array}$ & $\begin{array}{l}123.47 \pm 12.56 \\
83.96 \pm 8.44^{* \Delta}\end{array}$ & $\begin{array}{c}9.61 \pm 1.67 \\
4.54 \pm 1.22^{* \Delta}\end{array}$ & $\begin{array}{c}8.97 \pm 1.54 \\
4.26 \pm 0.73^{* \Delta}\end{array}$ & $\begin{array}{c}433.74 \pm 52.67 \\
236.71 \pm 39.82^{* \Delta}\end{array}$ \\
\hline Control group & $\begin{array}{c}\text { Before treatment } \\
\text { After treatment }\end{array}$ & $\begin{array}{l}122.76 \pm 12.41 \\
99.42 \pm 9.26^{*}\end{array}$ & $\begin{array}{l}9.58 \pm 1.72 \\
6.87 \pm 1.53^{*}\end{array}$ & $\begin{array}{c}8.83 \pm 1.45 \\
5.64 \pm 0.89^{*}\end{array}$ & $\begin{array}{c}430.95 \pm 53.74 \\
314.85 \pm 42.36^{*}\end{array}$ \\
\hline$X^{2}$ & & 11.124 & 6.173 & 8.265 & 13.814 \\
\hline$P$ & & $<0.01$ & $<0.01$ & $<0.01$ & $<0.01$ \\
\hline
\end{tabular}

Compared with the same group before treatment, ${ }^{*} P<0.01$; compared with the control group after treatment, ${ }^{\Delta} P<0.01$.
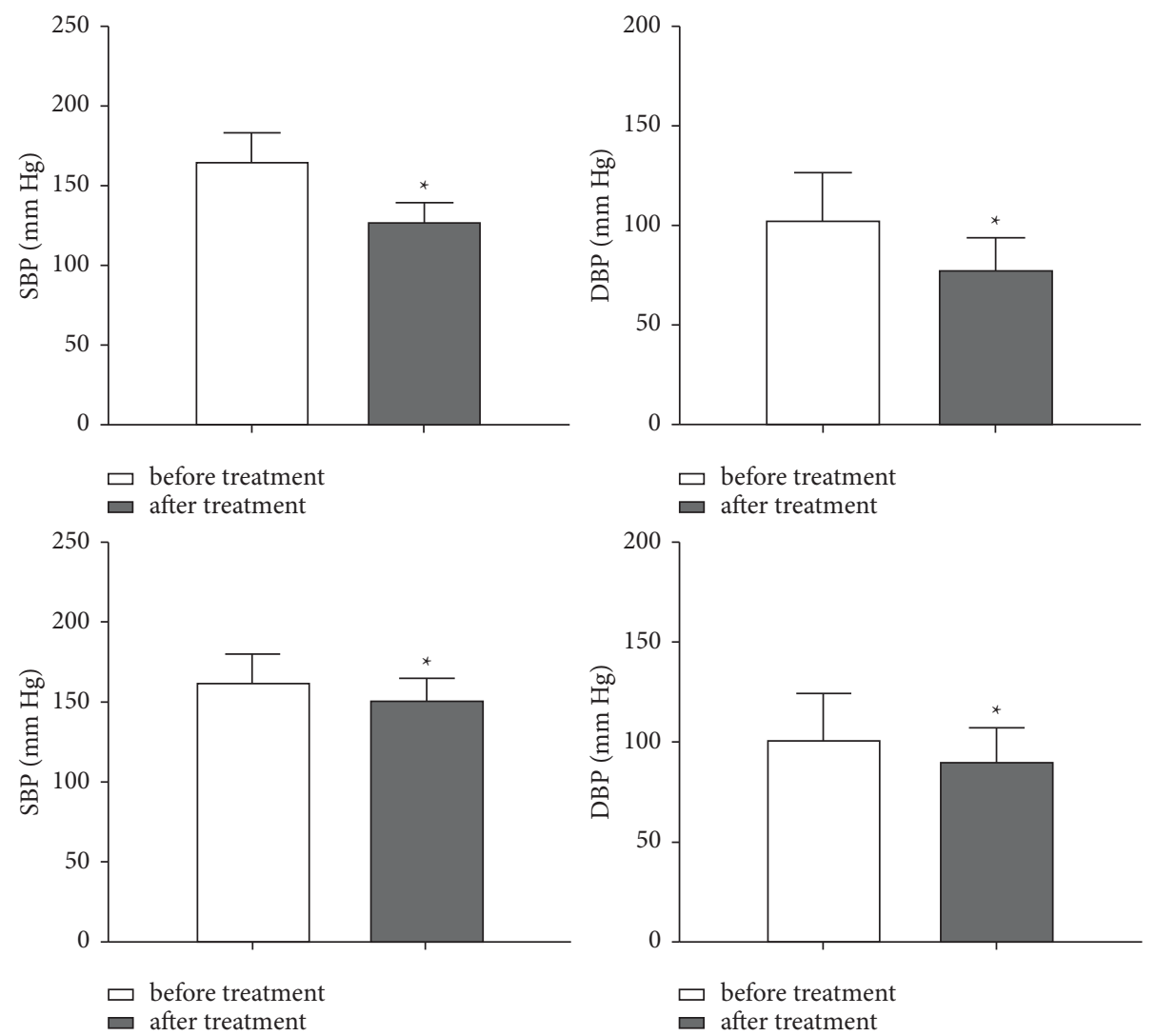

Figure 1: Comparison of the blood pressure between the two groups. (a) SBP in the experimental group was compared before and after treatment. (b) DBP in the experimental group was compared before and after treatment. (c) SBP in the control group was compared before and after treatment. (d) DBP in the control group was compared before and after treatment. ${ }^{*} P<0.05$.

phenomena. Severe cases are forced to terminate pregnancy or even die, which poses a serious threat to the life safety of pregnant women and fetus $[12,13]$. Through effective intervention to control pregnant women's blood pressure, the occurrence of cardiovascular and cerebrovascular diseases can be avoided, which is of great significance to ensure the healthy development of the fetus and guarantee the life safety of pregnant women. At present, a single antihypertensive drug combined with lifestyle improvement is used to treat patients with PIH, but the treatment effect is not ideal [14]. Due to the poor physical quality of pregnant women, this situation is easy to cause adverse effects in the fetus $[15,16]$. Labetalol hydrochloride is one of the antihypertensive drugs commonly used in the treatment of PIH, which can dilate blood vessels, reduce cardiac load and myocardial oxygen consumption, and increase cardiac output, so as to achieve the purpose of lowering blood pressure [17, 18]. Magnesium sulfate has the antagonistic effect of $\mathrm{Ca}^{2+}$ and is mainly used for anticonvulsion. It can reduce blood pressure by causing vascular dilation, further improve human microcirculation, and can be used for the treatment of hypertensive crisis $[19,20]$.

According to the traditional Chinese medicine system, due to the pregnancy of the fetus in the pregnant woman, all organs of the body cannot function normally, which will lead to the deficiency of the spleen and kidney yang deficiency, resulting in the disorder of water and moisture functions in the body [21]. Kidney yang deficiency leads to poor water flow in pregnant women and obstruction of fetal gas, resulting in the fetal and maternal deficiency of blood. 

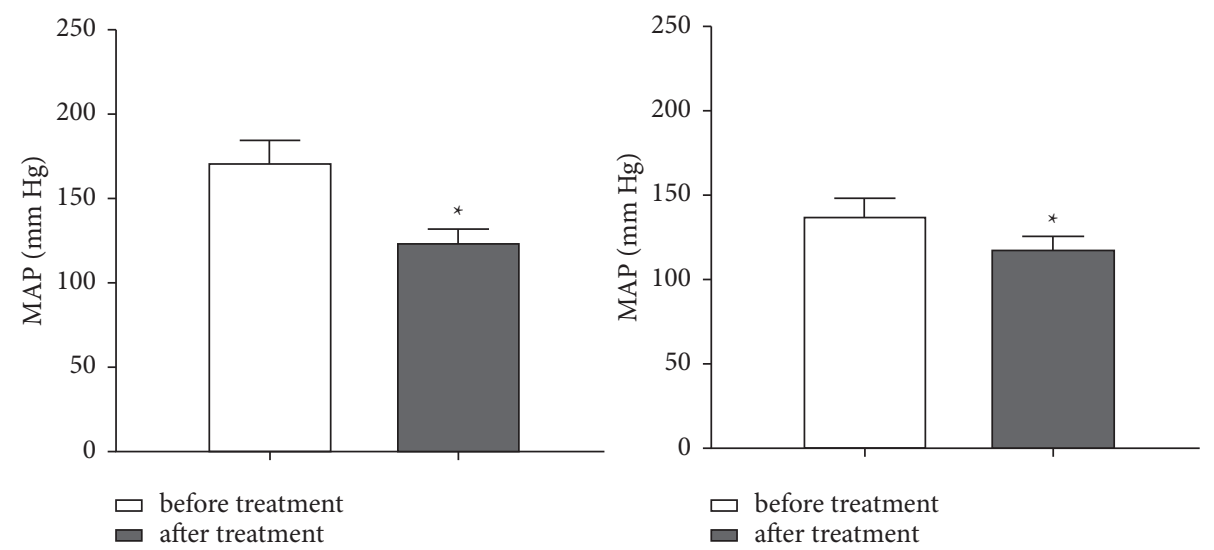

FIgURE 2: Comparison of the mean arterial blood pressure between the two groups. (a) MAP in the experimental group was compared before and after treatment. (b) MAP in the control group was compared before and after treatment. ${ }^{* *} P<0.05$.

TABLE 5: Comparison of the incidence of adverse reactions between two groups.

\begin{tabular}{lcccccc}
\hline Group & $n$ & Headaches & Nausea & Swelling & Vomiting & Muscle weakness \\
\hline Experimental group & 63 & 5 & 3 & 2 & 4 & 7 \\
Control group & 63 & 8 & 4 & 3 & 7 & 9 \\
$X^{2}$ & & 0.513 & 0.207 & 0.161 & 0.446 & 0.737 \\
$P$ & & $>0.05$ & $>0.05$ & $>0.05$ & $>0.05$ & $>0.05$ \\
\hline
\end{tabular}

TABLE 6: Comparison of pregnancy outcomes between the two groups after treatment.

\begin{tabular}{lccccccc}
\hline Group & $n$ & $\begin{array}{c}\text { Caesarean } \\
\text { section }\end{array}$ & $\begin{array}{c}\text { Premature } \\
\text { birth }\end{array}$ & $\begin{array}{c}\text { Fetal } \\
\text { distress }\end{array}$ & $\begin{array}{c}\text { Postpartum } \\
\text { hemorrhage }\end{array}$ & $\begin{array}{c}\text { Abnormal fetal heart } \\
\text { rate }\end{array}$ & $\begin{array}{c}\text { Neonatal } \\
\text { asphyxia }\end{array}$ \\
\hline $\begin{array}{l}\text { Experimental } \\
\text { group }\end{array}$ & 63 & 23 & 11 & 2 & 2 & 3 & 3 \\
Control group & 63 & 42 & 26 & 6 & 8 & 15 & 7 \\
$\mathrm{X}^{2}$ & & 5.623 & 4.216 & 0.451 & 0.782 & $>067$ & 0.486 \\
$P$ & $<0.05$ & $<0.05$ & $>0.05$ & $>0.05$ & $<0.05$ & $>0.05$ \\
\hline
\end{tabular}

Therefore, patients with PIH should tonify the kidney and qi [22]. In this paper, we study a TCM prescription of Pinggan Yiqi Yangshen recipe. Among them, Astragali Radix has the functions of tonifying qi, replenishing wei, and consolidating the exterior. Radix Codonopsis has the functions of tonifying qi and nourishing blood. Cortex Phellodendri and Gardenia jasminoides have the effects of clearing away heat, purging fire and dampness, and detoxification. Paeoniae Alba Radix and Cyperus rotundus have the effects of nourishing blood, softening the liver, and relieving pain. Pinelliae Rhizoma can dry wet and reduce phlegm. Citri Reticulatae Pericarpium has the function of regulating qi and dispelling phlegm. Atractylodes macrocephala and Poria $\operatorname{cocos}$ have the functions of moistening water, invigorating the spleen and stomach, and relieving the heart and placenta. Curcumae Radix and Angelica have the actions of supplementing blood, promoting blood circulation, and opening the meridians. Chrysanthemum morifolium and Semen Cassiae have the actions of calming liver wind. Bupleurum chinense, Angelica dahurica, and Uncaria rhynchophylla have the functions of relieving liver depression, calming liver wind, reinforcing qi, and elevating yang. Rhizoma Alismatis has the effect of inducing diuresis to alleviate edema. Eucommia ulmoides, donkey-hide gelatin, and Herba Taxilli have the actions of eliminating dampness, calming the liver, reinforcing the kidney, and preventing miscarriage. Citri Reticulatae Pericarpium has the functions of invigorating the spleen and regulating the stomach. The whole prescription can reinforce qi, strengthen the spleen, calm the liver, and reinforce the kidney [23-28]. Recent research has reported that Atractylodes macrocephala and Poria cocos can enhance human immunity [29, 30]. Uncaria rhynchophylla and Eucommia ulmoides have strong antihypertensive, sedative, spasmodic, and diuretic effects [31, 32]. Chrysanthemum morifolium extract solution can dilate blood vessels, reduce blood pressure, and reduce the cardiac output [33]. On the basis of western medicine use in hypertension, Chinese medicine can improve the possible adverse outcomes during pregnancy, improve human microcirculation, and can achieve the safe hypotension effect.

This study showed that, after treatment, both groups had certain clinical treatment effects. Our results showed that the combination of traditional Chinese and western medicine could effectively reduce the blood pressure and improve the 
clinical symptoms of pregnancy hypertension. After treatment, the levels of BUN, UA, Cr, and SU in the experimental group were lower than those in the control group. We identified that this was closely related to the efficacy of Astragali Radix, Herba Taxilli, and Eucommia ulmoides in these prescriptions, such as nourishing qi and nourishing yuan, tonifying the kidney, calming the liver, and strengthening body resistance to eliminate pathogenic factors. Pinggan Yiqi Yangshen recipe can improve the renal blood circulation and increase the blood flow, and it significantly decreased the level of renal function indicators. After treatment, the incidences of cesarean section, premature birth, and abnormal fetal heart rate in the experimental group were significantly higher than those in the control group. However, there was no difference in the incidence of fetal distress, postpartum hemorrhage, and neonatal asphyxia between the two groups, which may be related to the small number of cases included. NO, an important diastolic factor produced by endothelial cells, is a new type of transport molecule with free radical properties with the effect of vascular dilation. SOD is an antioxidant enzyme to defend against the damage and destruction of oxygen free radical in metabolism and other life activities. MDA is a major metabolite reflecting lipid peroxidation in the body. The level of MDA in plasma can reflect the content of free radicals in tissues and the degree of lipid peroxidation damage. ET-1 exists in vascular endothelial cells and is one of the most vital vasoconstrictor factors. Plasma ET-1 is associated with hemodynamic changes during normal pregnancy. ET-1 showed no significant difference in the first and second trimester, but increased significantly in the third trimester, interacting with other vasoactive substances to regulate blood pressure and hemodynamic changes [34-37]. In this study, the levels of NO and SOD increased in both groups after treatment, and the experimental group was significantly higher than the control group. The levels of MDA, RT-1, and Upro in the experimental group were significantly lower than those in the control group. The results showed that the combination of integrated Chinese and western medicine is effective in treating PIH and can adjust the level of each index to the normal range.

In conclusion, Pinggan Yiqi Yangshen recipe combined with labetalol hydrochloride and magnesium sulfate can effectively improve the clinical symptoms of PIH patients, restore the damaged liver and kidney function, and improve the adverse outcomes during pregnancy in the mother and infant, which is worthy of further clinical promotion.

\section{Data Availability}

The data used to support the findings of this study are available from the corresponding author upon request.

\section{Conflicts of Interest}

The authors declare that they have no conflicts of interest.

\section{Authors' Contributions}

Ping Li and Jie Zhao contributed equally to this work.

\section{References}

[1] E. Kintiraki, S. Papakatsika, G. Kotronis, D. Goulis, and V. Kotsis, "Pregnancy-Induced hypertension," Hormones, vol. 14, no. 2, pp. 211-223, 2015.

[2] K. Klipstein-Grobusch, "Systematic review of prediction models for gestational hypertension and preeclampsia," PLoS One, vol. 15, no. 4, Article ID e0230955, 2020.

[3] M. Watanabe, T. Sairenchi, K. Nishida et al., "Gestational hypertension as risk factor of hypertension in middle-aged and older women," International Journal of Environmental Research and Public Health, vol. 17, no. 11, Article ID 4052, 2020.

[4] Y. Wang, X. Zhang, Y. Han, F. Yan, and R. Wu, "Efficacy of combined medication of nifedipine and magnesium sulfate on gestational hypertension and the effect on PAPP-A, VEGF, NO, Hcy and vWF," Saudi Journal of Biological Sciences, vol. 26, no. 8, pp. 2043-2047, 2019.

[5] T. Easterling, S. Mundle, H. Bracken et al., "Oral antihypertensive regimens (nifedipine retard, labetalol, and methyldopa) for management of severe hypertension in pregnancy: an open-label, randomised controlled trial," The Lancet, vol. 394, no. 10203, pp. 1011-1021, 2019.

[6] K. Sridharan and R. P. Sequeira, "Drugs for treating severe hypertension in pregnancy: a network meta-analysis and trial sequential analysis of randomized clinical trials," British Journal of Clinical Pharmacology, vol. 84, no. 9, pp. 1906-1916, 2018.

[7] X.-F. Wang and M.-Q. Zhao, "Ligustrazine and Salvia miltiorrhiza injection solution in complementary therapy of pregnancy-induced hypertension: clinical analysis of 60 cases," Di Yi Jun Yi Da Xue Xue Bao, vol. 23, no. 9, pp. 969-971, 2003.

[8] P.-R. Yang, W.-T. Shih, Y.-H. Chu, P.-C. Chen, and C.-Y. Wu, "Frequency and co-prescription pattern of Chinese herbal products for hypertension in Taiwan: a Cohort study," BMC Complementary and Alternative Medicine, vol. 15, no. 1, p. $163,2015$.

[9] J. L. Hallock and V. L. Handa, "The epidemiology of pelvic floor disorders and childbirth," Obstetrics \& Gynecology Clinics of North America, vol. 43, no. 1, pp. 1-13, 2016.

[10] M. Shen, G. N. Smith, M. Rodger, R. R. White, M. C. Walker, and S. W. Wen, "Comparison of risk factors and outcomes of gestational hypertension and pre-eclampsia," PLoS One, vol. 12, no. 4, Article ID e0175914, 2017.

[11] C. Liu, C. Liu, Q. Wang, and Z. Zhang, "Supplementation of folic acid in pregnancy and the risk of preeclampsia and gestational hypertension: a meta-analysis," Archives of Gynecology and Obstetrics, vol. 298, no. 4, pp. 697-704, 2018.

[12] A. G. Kattah and V. D. Garovic, "The management of hypertension in pregnancy," Advances in Chronic Kidney Disease, vol. 20, no. 3, pp. 229-239, 2013.

[13] A. Dobrosavljevic and S. Rakic, "Risk of gestational hypertension in pregnancies complicated with ovarian hyperstimulation syndrome," JPMA. The Journal of the Pakistan Medical Association, vol. 70, no. 11, pp. 1897-1900, 2020.

[14] L. M. Webster, F. Conti-Ramsden, P. T. Seed, A. J. Webb, C. Nelson-Piercy, and L. C. Chappell, "Impact of antihypertensive treatment on maternal and perinatal outcomes in pregnancy complicated by chronic hypertension: a systematic review and meta-analysis," Journal of the American Heart Association, vol. 6, no. 5, Article ID e005526, 2017.

[15] T. Firoz, L. Magee, K. MacDonell et al., "Oral antihypertensive therapy for severe hypertension in pregnancy and postpartum: a systematic review," BJOG: An International Journal of 
Obstetrics and Gynaecology, vol. 121, no. 10, pp. 1210-1218, 2014.

[16] A. Whelan, J. Izewski, C. Berkelhammer, J. Walloch, and H. H. Kay, "Labetalol-induced hepatotoxicity during pregnancy: a case report," American Journal of Perinatology Reports, vol. 10, no. 03, pp. e210-e212, 2020.

[17] M. F. Gaynor, G. C. Wright, and S. Vondracek, "Retrospective review of the use of as-needed hydralazine and labetalol for the treatment of acute hypertension in hospitalized medicine patients," Therapeutic Advances in Cardiovascular Disease, vol. 12, no. 1, pp. 7-15, 2018.

[18] P. Patel, D. Koli, N. Maitra, T. Sheth, and P. Vaishnav, "Comparison of efficacy and safety of intravenous labetalol versus hydralazine for management of severe hypertension in pregnancy," Journal of Obstetrics \& Gynaecology of India, vol. 68, no. 5, pp. 376-381, 2018.

[19] C. Xiang, X. Zhou, and X. Zheng, "Magnesium sulfate in combination with nifedipine in the treatment of pregnancyinduced hypertension," Pakistan Journal Of Medical Sciences, vol. 36, no. 2, pp. 21-25, 2020.

[20] G. He, Y. Chen, M. Chen, G. He, and X. Liu, "Efficacy and safety of low dose aspirin and magnesium sulfate in the treatment of pregnancy induced hypertension," Medicine, vol. 99, no. 46, Article ID e22801, 2020.

[21] F. Greenway, Z. Liu, Y. Yu, and A. Gupta, "A clinical trial testing the safety and efficacy of a standardized Eucommia ulmoides Oliver bark extract to treat hypertension," Alternative Medicine Review: A Journal of Clinical Therapeutic, vol. 16, no. 4, pp. 338-347, 2011.

[22] W. Wu, Z. Zhang, F. Li et al., "A network-based approach to explore the mechanisms of Uncaria alkaloids in treating hypertension and alleviating alzheimer's disease," International Journal of Molecular Sciences, vol. 21, no. 5, Article ID 1766, 2020.

[23] Y. Liu, M. Xiong, F. Zhou, N. Shi, and Y. Jia, "Effect of baicalin on gestational hypertension-induced vascular endothelial cell damage," Journal of International Medical Research, vol. 48, no. 10, 2020.

[24] T. Hussain, B. Tan, G. Liu et al., "Health-promoting properties of Eucommia ulmoides: a review," Evidence-Based Complementary and Alternative Medicine, vol. 2016, Article ID 5202908, 9 pages, 2016.

[25] Z. Tian, S. Zhang, H. Wang et al., "Intervention of Uncaria and its components on liver lipid metabolism in spontaneously hypertensive rats," Frontiers in Pharmacology, vol. 11, p. 910, 2020.

[26] Q. Xing, L. Fu, Z. Yu, and X. Zhou, "Efficacy and safety of integrated traditional Chinese medicine and western medicine on the treatment of rheumatoid arthritis: a meta-analysis," Evidence-based Complementary and Alternative Medicine, vol. 2020, Article ID 4348709, 15 pages, 2020.

[27] M. H. Yeh, H. P. Chiu, M. C. Wu et al., "Integrated Chinese herbal medicine and western medicine on the survival in patients with colorectal cancer: a retrospective study of medical records," Evidence-Based Complementary and Alternative Medicine, vol. 2020, Article ID 4561040, 10 pages, 2020.

[28] M. Heng, J. Tu, Y. Hao et al., "Effects of integrated traditional Chinese and western medicine for the treatment of lupus nephritis: a meta-analysis of randomized trials," EvidenceBased Complementary and Alternative Medicine, vol. 2016, Article ID 1502107, 7 pages, 2016.

[29] S. Zhang, L. Zhang, K. Long et al., "Evaluation of clinical efficacy of integrated traditional Chinese and Western medicine in the treatment of acute respiratory distress syndrome," Medicine, vol. 99, no. 25, Article ID e20341, 2020.

[30] Y. Meng, T. M. Michelena, F. Cai, X. Lou, S. Li, and R. Zhang, "Traditional Chinese medicine in emergency treatment mechanism and application," Open Access Emergency Medicine, vol. 12, pp. 111-119, 2020.

[31] D. Zhen-Jiang, L. Chao, W. Xiao et al., "Antihypertensive activity of Eucommia ulmoides oliv: male flower extract in spontaneously hypertensive rats," Evidence-Based Complementary and Alternative Medicine, vol. 2020, Article ID 6432173, 6 pages, 2020

[32] M. Sun, H. Wang, L. Gong et al., "A novel time-dimension and circadian rhythm-dependent strategy for pharmacodynamic evaluation of Uncaria in the regulation of neurotransmitter circadian metabolic homeostasis in spontaneously hypertensive rats," Biomedicine \& Pharmacotherapy, vol. 131, Article ID 110704, 2020.

[33] F. S. Youssef, S. Y. Eid, E. Alshammari, M. L. Ashour, M. Wink, and M. Z. El-Readi, "Chrysanthemum indicum and Chrysanthemum morifolium: chemical composition of their essential oils and their potential use as natural preservatives with antimicrobial and antioxidant activities," Foods, vol. 9, no. 10, Article ID 1460, 2020.

[34] H. X. Guo, Y. B. Zhu, C. P. Wu, M. Zhong, and S. W. Hu, "Potential urine biomarkers for gestational hypertension and preeclampsia," Molecular Medicine Reports, vol. 19, no. 4, pp. 2463-2470, 2019.

[35] L. Zhang, J. Sun, M. Zhang et al., "The significance of combined detection of $\mathrm{CysC}$, urinary $\mathrm{mAlb}$ and $\beta 2-\mathrm{MG}$ in diagnosis of the early renal injury in pregnancy-induced hypertension syndrome," Saudi Journal of Biological Sciences, vol. 26, no. 8, pp. 1982-1985, 2019.

[36] J. Ainuddin, F. Javed, and S. Kazi, "Oral labetalol versus oral nifedipine for the management of postpartum hypertension a randomized control trial," Pakistan journal of medical sciences, vol. 35, no. 5, pp. 1428-1433, 2019.

[37] Y. Zeng, M. Li, Y. Chen, and S. Wang, "Homocysteine, endothelin-1 and nitric oxide in patients with hypertensive disorders complicating pregnancy," International Journal of Clinical and Experimental Pathology, vol. 8, no. 11, pp. 15275-15279, 2015. 